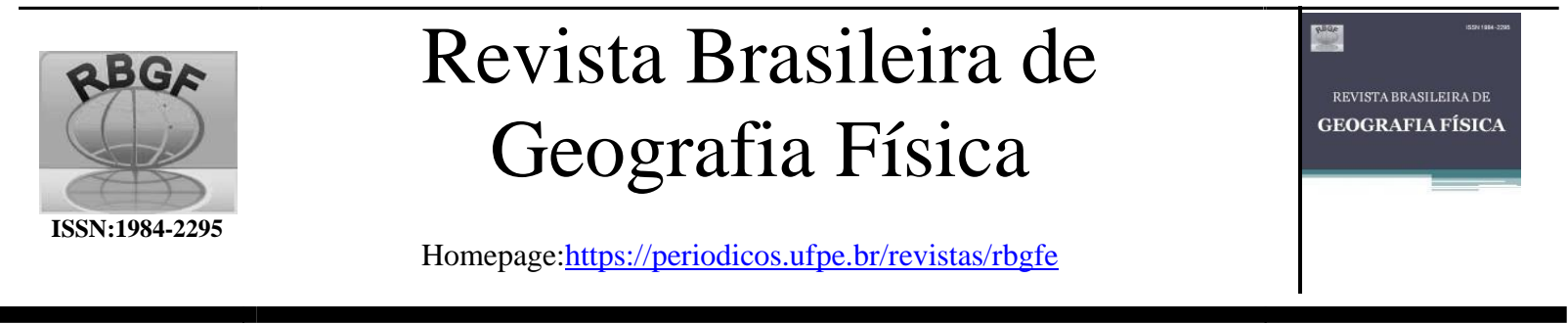

\title{
Análise da dinâmica do uso e cobertura da terra do Município de Moju- PA, utilizando Google Earth Engine
}

Samuel Salin Gonçalves de Souza ${ }^{1}$, Jones Remo Barbosa Vale ${ }^{2}$, Merilene do Socorro Silva Costa ${ }^{3}$, Bruna Ribeiro Chagas $^{4}$, Carolina da Silva Gonçalves ${ }^{5}$, Matheus Gabriel Lopes Botelho ${ }^{6}$, Layse Gomes Furtado ${ }^{7}$, Carla Renata de Oliveira Carneiro ${ }^{8}$, Vanessa de Almeida Batista ${ }^{9}$, Gundisalvo Piratoba Morales ${ }^{10}$

\begin{abstract}
'Graduando de Engenharia Cartográfica e de Agrimensura, Universidade Federal Rural da Amazônia, Av. Tancredo Neves, 2501, - CEP: 66.077-830, Belém-PA. (91) 99203-9281. samuelsalin16@gmail.com. ${ }^{2}$ Ms. e Doutorando em Geografia, Universidade Federal do Pará, Rua Augusto Corrêa, 01, CEP: 66075-110, Belém-PA. (91) 3201-7390. jonesremo@ hotmail.com. ${ }^{3}$ Professora Dra. Adjunto IV, Universidade Federal Rural da Amazônia, Av. Tancredo Neves, 2501, - CEP: 66.077-830, Belém-PA. (91) 99203-9281. merilene@ @otmail.com. ${ }^{4}$ Graduanda de Engenharia Cartográfica e de Agrimensura, Universidade Federal Rural da Amazônia, Av. Tancredo Neves, 2501, - CEP: 66.077-830, Belém-PA. (91) 99203-9281. bruna.chagas92@gmail.com. ${ }^{5}$ Engenheira Cartográfica e de Agrimensura pela Universidade Federal Rural da Amazônia (UFRA). Mestranda do Programa de Pós-Graduação em Geografia da Universidade Federal do Pará (UFPA), Rua Augusto Corrêa, 01, CEP: 66075-110, Belém-PA. (91) 32017390. linacrsg@ gmail.com. ${ }^{6}$ Engenheiro Agrônomo pela Universidade Federal Rural da Amazônia. Mestrando em Ciências Ambientais - Programa de Pós-Graduação em Ciências Ambientais - Universidade do Estado do Pará, Tv. Dr. Enéas Pinheiro, 2626, CEP 56900-000, Belém, Pará. (91) 31311914. math.botelho2194@gmail.com (autor correspondente). ${ }^{7}$ Especialista em Geoprocessamento aplicado à Agroecologia e ao Uso de Recursos Naturais pela Universidade Federal do Pará. Mestranda em Ciências Ambientais - Programa de Pós-Graduação em Ciências Ambientais - Universidade do Estado do Pará, Tv. Dr. Enéas Pinheiro, 2626, CEP 56900-000, Belém, Pará. (91) 3131-1914. layse.furtadog@ gmail.com. ${ }^{8}$ Licenciada em Ciências Naturais com habilitação em Biologia. Mestranda do Programa de Pós-Graduação em Ciências Ambientais (PPGCA) - Universidade do Estado do Pará, Tv. Dr. Enéas Pinheiro, 2626, CEP 56900-000, Belém, Pará. (91) 3131-1914. carlacarneiro7@ outlook.com. ${ }^{9}$ Bióloga pela Universidade Federal do Rio de Janeiro (UFRJ). Mestranda do Programa de Pós-Graduação em Ciências Ambientais (PPGCA) - Universidade do Estado do Pará, Tv. Dr. Enéas Pinheiro, 2626, CEP 56900-000, Belém, Pará. (91) 3131-1914. vanessavab @ hotmail.com. ${ }^{10}$ Professor Dr. Adjunto IV, Universidade do Estado do Pará, Tv. Dr. Enéas Pinheiro, 2626, CEP 56900-000, Belém, Pará. (91) 3131-1914. gundymorales@ gmail.com.
\end{abstract}

Artigo recebido em 12/06/2020 e aceito em 12/10/2020.

R E S U M O

Em razão das grandes transformações na paisagem ocasionadas pelas principais atividades econômicas do município de Moju, como as práticas agropecuárias, esta pesquisa procurou fazer uma análise temporal e espacial das mudanças de uso e cobertura da terra na localidade, nos anos de 1999 e 2018, por meio de imagens de satélite disponibilizadas pela plataforma Google Earth Engine. Utilizou-se imagem do satélite Landsat-5/TM referente ao ano 1999, e imagem do satélite Landsat-8/OLI-TIRS correspondente ao ano de 2018, ambas disponíveis no Google Earth Engine (GEE). Realizou-se a classificação temporal e espacial de uso e cobertura da terra por meio da aplicação do algoritmo Random Forest. Utilizou-se as análises qualitativas e quantitativas para os dados mapeados, com o objetivo de realizar um detalhamento sobre a dinâmica do uso e cobertura da terra por meio de tabela e mapa. Os resultados apontam que houve uma perda de mais de $878 \mathrm{~km}^{2}$ de cobertura vegetal correspondendo cerca de $12 \%$ de perda que veio em decorrência das atividades antrópicas que ocorreram em Moju, principalmente, em relação à agricultura com os cultivos de dendê, mandioca, coco e à pecuária com as áreas de pastagens, pois, juntos apresentam um aumento de mais de mais de $70 \%$ que equivalem a 1.037,0938 km². Portanto, constatou-se que o desenvolvimento econômico do município de Moju segue o padrão de desenvolvimento dos municípios amazônicos, onde ocorre a diminuição das áreas florestais para a expansão de suas atividades produtivas, como os cultivos de dendê, sendo este um dos principais indutores do desflorestamento no município.

Palavras-Chave: desmatamentos, atividades agropecuárias, transformações na paisagem.

\section{Analysis of the dynamics of the use and land coverage of the Municipality of Moju-PA, using the Google Earth Engine}

\begin{abstract}
A B S T R A C T
Due to the great transformations in the landscape caused by the main economic activities of the municipality of Moju, such as agricultural practices, this research sought to make a temporal and spatial analysis of changes in land use and coverage in the locality, in the years 1999 and 2018, by through satellite images made available by the Google Earth
\end{abstract}


Engine platform. An image of the Landsat-5 / TM satellite for the year 1999 was used, and an image of the Landsat-8 / OLI-TIRS satellite for the year 2018, both available on the Google Earth Engine (GEE). The temporal and spatial classification of land use and land cover was carried out by applying the Random Forest algorithm. Qualitative and quantitative analyzes were used for the mapped data, with the aim of detailing the dynamics of land use and land cover using a table and map. The results show that there was a loss of more than $878 \mathrm{~km}^{2}$ of vegetation cover, corresponding to about $12 \%$ of the loss that came as a result of the anthropic activities that occurred in Moju, mainly, in relation to agriculture with oil palm, cassava, coconut and livestock with pasture areas, because together they show an increase of more than more than $70 \%$, which is equivalent to $1,037.0938 \mathrm{~km}^{2}$. Therefore, it was found that the economic development of the municipality of Moju follows the pattern of development of the Amazonian municipalities, where there is a decrease in forest areas for the expansion of their productive activities, such as oil palm cultivation, which is one of the main drivers of the deforestation in the municipality.

Keywords: deforestation; agricultural activities; transformations in the landscape.

\section{Introdução}

A pesquisa sobre as estruturas que integram o estudo da dinâmica das paisagens possui potencial para promover conhecimentos técnicos que são capazes de apoiar medidas eficazes no que se refere ao planejamento da gestão territorial e ambiental, assim como, compreender a relação da sociedade com a cobertura da terra. Nesse contexto, se destaca o município de Moju (Pará) por ser uma localidade amazônica que se enquadra no panorama de transformações antrópicas na paisagem natural.

A região Amazônica vem passando por intensas mudanças de uso e cobertura da terra. Essas modificações na paisagem local são resultantes de uma série de fatores, tais como: questões macroeconômicas envolvendo a exploração madeireira, a pecuária (Mertens et al., 2002), cultivos agrícolas e abertura de estradas (Nepstad et al., 2000).

O mapeamento da dinâmica do uso e cobertura da terra torna-se fundamental para auxiliar no planejamento ambiental, principalmente com fornecimento de dados que subsidiam o desenvolvimento de políticas no que tange o monitoramento do desflorestamento, pois, permite analisar o vetor de expansão urbana e auxiliar na fiscalização de atividades ilegais de mineração, ou seja, com o mapeamento de uso e cobertura identificam-se as atividades antrópicas e os problemas ambientais que elas provocam, contribuindo então para mitigar e solucionar tais problemas (Steffens et al., 2004).

Nesse contexto, as ferramentas de geoprocessamento são caracterizadas como um conjunto de aparatos tecnológicos que integram análise de dados, processamento e a coleta, uma vez que os mesmos são capazes de investigar sobre a dinâmica de cobertura da terra a nível local, regional ou municipal (Gollnow et al., 2014). Dessa forma, o mapeamento adequado favorece o controle e o planejamento que possuem como finalidade a gestão sustentável de recursos naturais (Almeida et al., 2014).
Nesses mapeamentos são utilizadas geotecnologias, que são tecnologias espaciais modernas (Vale, 2019). Recentemente surgiu uma nova geotecnologia que funciona como plataforma online que detém de uma série de dados ambientais globais, capazes de auxiliar no desenvolvimento de pesquisas e análises de uso e cobertura da terra. Essa plataforma é o Google Earth Engine (GEE). Segundo Gorelick et al. (2017), o GEE é uma plataforma composta por um catálogo de várias imagens de satélites e conjuntos de dados geoespaciais, que vem auxiliando em mapeamentos em escala global visando, principalmente análises de dados ambientais.

Para a maior discussão sobre as mudanças antrópicas nos ambientes, surge o seguinte questionamento: qual a relação das atividades econômicas com o desmatamento e o crescimento das áreas antropizadas? Para isso, o presente trabalho objetiva analisar espacialmente e temporalmente o uso e cobertura do solo do município de Moju-PA, afim de proporcionar o maior entendimento da dinâmica das paisagens.

\section{Material e Métodos}

Área de estudo

O município de Moju (Figura 1) localizase na região intermediária de Belém, no Estado do Pará, limitando-se ao norte com os municípios de Barcarena e Belém, ao leste com Tailândia e Acará, ao sul com Breu Branco e a oeste com Mocajuba, Igarapé-Miri e Abaetetuba possuindo uma área de $9.094,135 \mathrm{~km}^{2}$. De acordo com IBGE (2015), a população da localidade corresponde a 77.385 habitantes. Segundo a classificação de Köppen, o clima da região é Ami (quente e úmido), apresentando temperatura média anual em torno de $25,5^{\circ} \mathrm{C}$. Quanto à umidade relativa do ar, a cidade apresenta um valor aproximado de $85 \%$. A maior taxa de pluviosidade corresponde ao período entre os meses de janeiro a junho, ocasionando uma precipitação pluviométrica de 2.000 a 3.000 $\mathrm{mm}$ /ano (Modesto Junior et al., 2008). Os cultivos 
das culturas agrícolas, como o açaí (Euterpe oleracea Mart.), coco (Cocos nucifera L.), dendê (Elaeis guineensis Jacq.) e a mandioca (Manihot esculenta Crantz), além da produção de carvão, extração de madeira e as práticas de pecuária, representam a economia do município (IBGE, 2015).

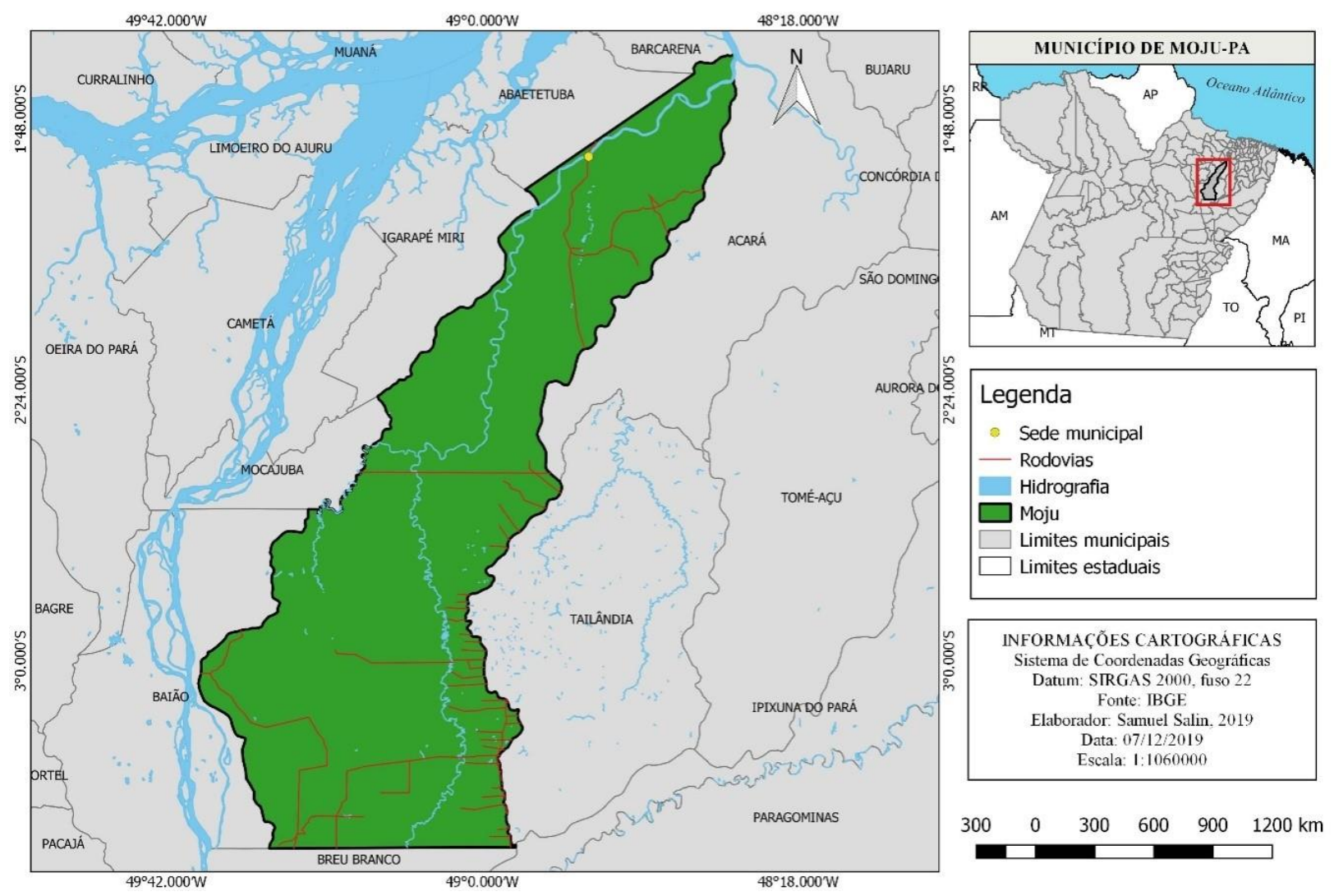

Figura 1. Mapa de localização do município de Moju.

Aquisição, processamento e análise de dados

A pesquisa incluiu três etapas: A primeira se baseou em revisão de literatura no Portal de Periódicos da Capes e no Portal Scielo sobre a dinâmica do uso e cobertura da terra em localidades amazônicas, como o município de Moju.

Na segunda, houve a realização da análise das mudanças na dinâmica de uso e cobertura da terra no município de Moju. Utilizou-se imagem do satélite Landsat-5/TM referente ao ano 1999, e imagem do satélite Landsat-8/OLI-TIRS correspondente ao ano de 2018, ambas disponíveis no Google Earth Engine (GEE). Esta ferramenta é uma plataforma avançada que realiza um geoprocessamento espacial fundamentado em nuvem, com finalidade de análise de dados de cunho ambiental em proporção planetária. Este dispositivo possibilita uma manipulação espacial realizada por pesquisadores, para descobrir e quantificar transformações sobre a superfície da Terra. Assim como, favorece estudos sobre mapas de tendência (Gorelick et al., 2017).

$\mathrm{Na}$ terceira etapa, realizou-se a classificação temporal e espacial de uso e cobertura da terra por meio da aplicação do algoritmo Random Forest, pois, segundo Gorelick et al. (2017), é um classificador do tipo árvores de decisão, que por um conjunto de treinamento original realiza a classificação em meios estatísticos. As características mapeadas estão dispostas no Quadro 1. Utilizou-se as análises qualitativas e quantitativas para os dados mapeados, com o objetivo de realizar um detalhamento sobre a dinâmica do uso e cobertura da terra por meio de tabela e mapa. 
Quadro 1. Características das classes de uso e cobertura da terra

\begin{tabular}{|c|c|}
\hline Classe & \multicolumn{1}{|c|}{ Características } \\
\hline Área Antropizada & $\begin{array}{l}\text { Estradas pavimentadas e não-pavimentadas, solo } \\
\text { exposto, área agrícola, área de pasto, área de } \\
\text { mineração e ocupação urbana. }\end{array}$ \\
\hline Cobertura Vegetal & $\begin{array}{l}\text { Áreas com cobertura vegetal arbórea, arbustiva ou } \\
\text { gramínea, seja natural primária, regenerada ou em } \\
\text { processo de regeneração. }\end{array}$ \\
\hline Hidrografia & $\begin{array}{c}\text { Áreas com espelho d'água superficial, seja represa, } \\
\text { rio, igarapé ou córrego. }\end{array}$ \\
\hline Outros & $\begin{array}{l}\text { Nuvem, sombra de nuvem ou qualquer feição que } \\
\text { não se encaixe em alguma das classes anteriores. }\end{array}$ \\
\hline
\end{tabular}

\section{Resultados e Discussão}

O município de Moju, tem sido alvo de grandes transformações na paisagem, devido à ampliação das atividades produtivas, como as atividades agropecuárias, ocasionando a elevação dos níveis de desmatamentos na cidade (Cardoso et al, 2016). Sendo assim, o Ministério do Meio Ambiente (MMA) definiu o município como uma das localidades prioritárias em 2011 para combater questões relacionadas ao meio natural, de acordo com a Portaria $n^{\circ}$ 175/2011 (BRASIL, 2011). Diante desse panorama, as fiscalizações ambientais passaram a ser mais rígidas quanto às aplicabilidades das leis ambientais.

Dentre as atividades econômicas produtivas desenvolvidas no município de Moju, por empresas ou empresários individuais, estão as atividades madeireiras (extração e beneficiamento), extração de seixo, extrativismo de seringueira (Hevea brasiliensis L.), que acontecem no Projeto Seringa no Alto rio Moju; monocultura de coco (Cocos nucifera L.), localizado na empresa SOCOCO, e a monocultura de dendê (Elaeis guineensis Jacq.), do Grupo Agropalma, Marborges, Belém Bioenergia e Biopalma; e pecuária bovina e bubalina, às margens das rodovias PA-150 e PA-252 (Santos et al., 2017).

Nesse contexto, Pimenta et al. (2018), realizou um estudo sobre a análise da dinâmica da mudança de classes de uso da terra entre os anos de 2008 e 2014, no município de Moju (Pará). A metodologia utilizada se baseou na utilização dos Souza, S. S. G. de.; Vale, J. R. B.; Costa, M. do. S. S.; Chagas, B.R.; Gonçalves, C. S.; Botelho, M. G. L.; Furtado, L. G.; Carneiro, C. R. O.; Batista, V. A.; Morales, G. P. dados do projeto TerraClass, e utilizou técnicas de geoprocessamento para localizar espacialmente as principais classes de uso e cobertura do solo. A autora constatou que houve uma diminuição de desmatamentos na região, principalmente após o ano de 2010, porém, observou-se que houve um aumento de $22 \%$ das áreas de pastagens entre os anos estudados, assim como ocorreu uma tendência da utilização de agricultura anual realizada pelos agricultores da região. Dessa forma, percebe-se que ocorre no município um aumento das áreas antropizadas entre os anos de 2008 e 2014, embora o município tenha sido inserido na lista de localidades prioritárias para combater impactos ambientais pelo Ministério do Meio Ambiente, em 2011.

É notória uma indiferença e despreocupação dos agricultores que residem nos municípios amazônicos, uma vez que os mesmos continuam realizando práticas agrícolas insustentáveis, como a utilização de queimadas para a limpeza de áreas voltadas para a agricultura, assim como o uso de defensivos químicos prejudiciais ao meio ambiente. Programas de Educação Ambiental podem favorecer a implantação dos princípios de desenvolvimento sustentável nos modos de produção da Amazônia. Contudo, as práticas ambientalmente corretas precisam ser realizadas a partir da consciência dos próprios agricultores ou moradores de comunidades amazônicas (Almeida et al., 2014). Esforços das esferas governamentais (municipal, estadual e federal) têm sido realizados para 
diminuir as taxas de desmatamento no município de Moju (Pimenta et al., 2018).

$\mathrm{O}$ início do processo de inserção da dendeicultura na microrregião de Tomé-Açu provocou a dinâmica socioeconômica-ambiental, a reorganização da paisagem e a reconfiguração territorial, contribuindo para as modificações no uso e cobertura do solo em outras localidades, como o município de Moju, onde estar localizado um dos polos de plantações da empresa Biopalma. Tal instituição contribuiu para a integração de vários agricultores familiares aos projetos de produção do dendê, ocasionando uma reconfiguração no espaço rural. Dessa forma, verifica-se o alto índice de investimentos agrícolas no território amazônico paraense (Santos et al., 2017).

Segundo Bastos et al., (2010), o município de Moju apresenta uma dinâmica diferente, referente a população, comparada a outros municípios da região Tocantins, a população urbana de Moju, desde a década de 1980 tem sido maior do que a população rural. Estima-se uma taxa de urbanização de $34,69 \%$. De acordo com essa pesquisa, o município de Moju é o segundo maior produtor de dendê da região do Tocantins (24\%), apesar dessa cultura ser muito presente no município, a produção de culturas perenes, como o coco também ganha espaço, concentrando-se em $41 \%$ da área do município.

Em consonância com os dados de crescimento do PIB municipal, no período de 2003 a 2006, o município de Moju apresentou um crescimento anual médio positivo. O município é cortado por várias estradas de grande acesso, possibilitando uma crescente circulação de mercadoria e interligando vários mercados, o que contribui para o crescimento dessa taxa (Bastos et al., 2010). Segundo Santos et al., (2017), a abertura de estrada é um vetor que contribui para o fluxo financeiro, o qual passa a orbitar sobre produtos até então desconhecidos em uma localidade, o autor exemplifica o processo de queda relativa e absoluta predominância da produção de determinados produtos em comparação à ascensão de outros, como ocorreu com a produção de farinha e dendê no município de Moju. Entre os anos de 2003 a 2006, o município apresentou uma produção agropecuária mais diversificada e maiores áreas ocupadas com a produção agropecuária da região Tocantins. Esse setor cresceu 2,5\% ao ano, isso certamente reflete a migração tanto da indústria madeireira de outras regiões bem como parte da atividade pecuária para este município (Bastos et al., 2010).

De acordo com o IBGE (2018), o estado do Pará produziu 1.533.735 toneladas de dendê (cachos de coco), no ano de 2018. O município de Moju realizou a produção de 141.151 toneladas no mesmo ano. Dessa forma, a quantidade produzida no município correspondeu a 9,2\% em relação à produção realizada no Estado. Percebe-se que entre os anos de 2014 a 2018, a quantidade produzida do fruto no município de Moju permanece constante. No que se refere aos anos avaliados nesta pesquisa, observa-se que houve mudanças consideráveis quanto à quantidade produzida de dendê nos anos de 1999 e 2018. Nota-se que em 1999, o Estado do Pará produziu apenas 516.721 toneladas. O município de Moju efetuou 22.968 (Tabela 1). O aumento de produção do fruto no Estado e no município, nos anos mais atuais, acontece principalmente devido ao aumento de áreas destinadas ao cultivo de dendê, proporcionado pela integração da agricultura familiar local com as empresas de dendeicultura, ocasionando mudanças nos traços estruturais do território, no que se refere ao aumento de espaços agropecuários.

Tabela 1. Quantidade produzida de dendê no Estado do Pará e no município de Moju no ano de 1999 e entre os anos de 2014 a 2018.

\begin{tabular}{ccccccc}
\hline Estado e município & $\mathbf{1 9 9 9}$ & $\mathbf{2 0 1 4}$ & $\mathbf{2 0 1 5}$ & $\mathbf{2 0 1 6}$ & $\mathbf{2 0 1 7}$ & $\mathbf{2 0 1 8}$ \\
\hline Pará & 516.712 & 1.187 .338 & 1.379 .192 & 1.486 .082 & 1.634 .476 & 1.533 .735 \\
\hline Moju & 22.968 & 141.151 & 141.151 & 141.151 & 141.151 & 141.151 \\
\hline
\end{tabular}

Na Tabela 2, verifica-se que ocorre uma tendência de aumento de quantidades de áreas plantadas de dendê entre os anos de 2014 a 2018, no Estado do Pará. Quanto ao município de Moju, a quantidade de áreas plantadas permanece constante no mesmo período. Nota-se que o Estado do Pará apresentava 38.243 áreas em 1999. Em 2018, o Estado reteve 100.965 áreas. Em Moju, verifica-se que o mesmo teve 1.914 e 7.093 áreas plantadas nos anos de 1999 e 2018, respectivamente (IBGE, 2018). 
Tabela 2. Área plantada de dendê no Estado do Pará e no município de Moju no ano de 1999 e entre os anos de 2014 a 2018.

\begin{tabular}{ccccccc}
\hline Estado e município & $\mathbf{1 9 9 9}$ & $\mathbf{2 0 1 4}$ & $\mathbf{2 0 1 5}$ & $\mathbf{2 0 1 6}$ & $\mathbf{2 0 1 7}$ & \multirow{2}{*}{$\mathbf{2 0 1 8}$} \\
\hline Pará & 38.243 & 72.375 & 85.942 & 99.402 & 101.795 & 100.965 \\
\hline Moju & 1.914 & 7.093 & 7.093 & 7.093 & 7.093 & 7.093 \\
\hline
\end{tabular}

Diante da enorme demanda de produção de dendê, considerando um fator predominante para as mudanças socioespaciais do município, na Figura 2 e na Tabela 3 são apresentados os resultados da classificação de uso e cobertura da terra com base nas imagens de satélite de 1999 e 2018. Nota-se que em 1999, o município apresentava uma área de cobertura vegetal bem mais densa quando comparada a 2018. Dessa forma, cedendo espaço para as áreas antropizadas alterando a configuração da paisagem local mais recente. A cobertura vegetal cedeu espaço para o desenvolvimento das áreas agropecuárias que começaram se instalando nas margens das principais rodovias que cortam o município. Esse fato se evidencia na pesquisa de Almeida e Vieira (2014), pois, o município tem sido alvo de migração de empresas agrícolas, ocasionando um aumento na implantação de espaços voltados para a agricultura na localidade.

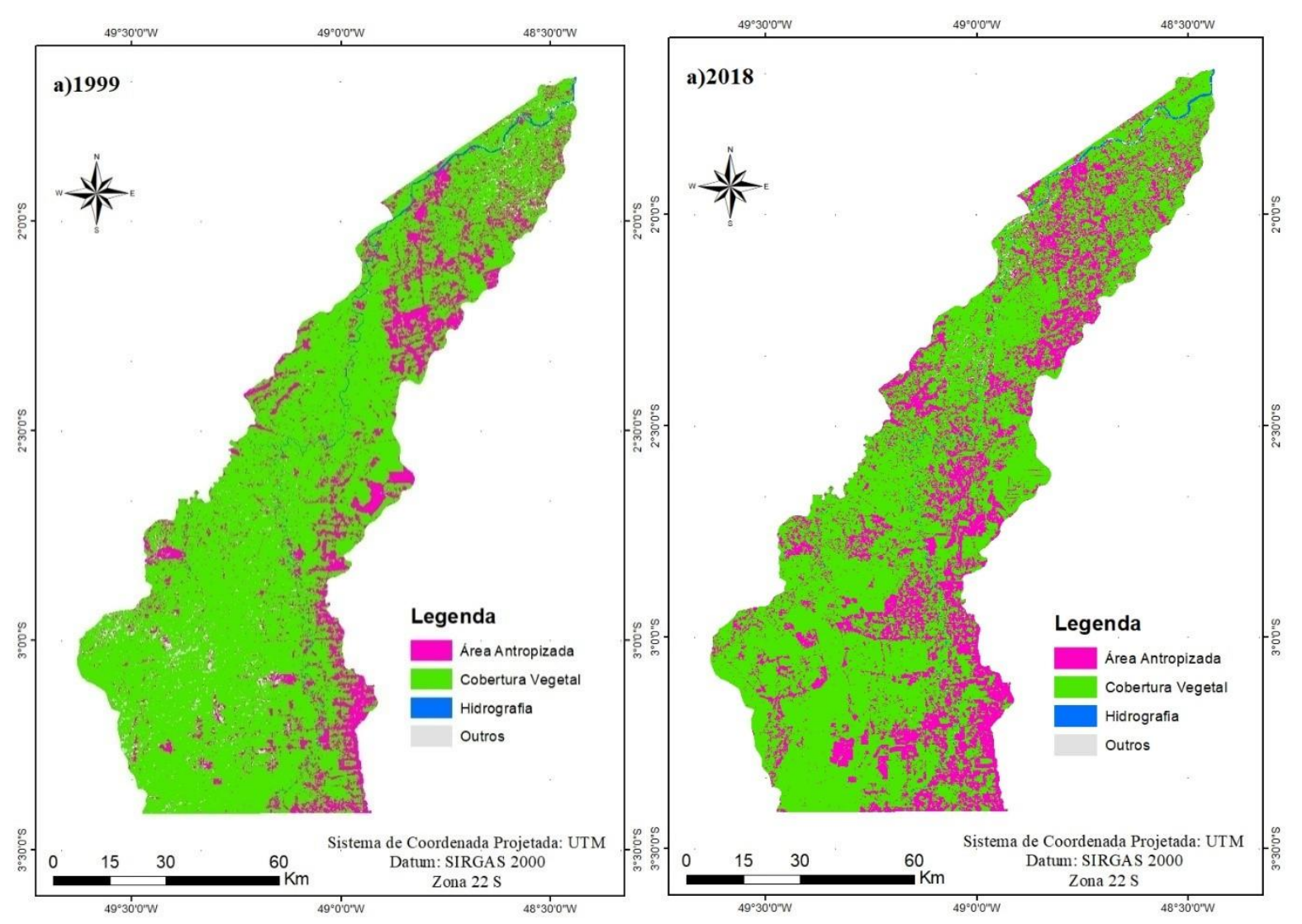

Figura 2. Classificação de uso e cobertura da terra do município de Moju em 1999 e 2018. 
Tabela 3. Valores em área $\left(\mathrm{km}^{2}\right)$ das classes de uso e cobertura da terra.

\begin{tabular}{ccc}
\hline CLASSE & $\mathbf{1 9 9 9}$ & $\mathbf{2 0 1 8}$ \\
\hline Área Antropizada & $1.468,6100$ & $2.505,7038$ \\
Cobertura Vegetal & $7.369,3400$ & $6.491,1033$ \\
Hidrografia & $65.172,3$ & $47.720,4$ \\
\hline Outros & $\mathbf{2 0 4 . 4 9 5 0}$ & $\mathbf{6 3 . 0 8 9 5}$ \\
\hline
\end{tabular}

Os resultados apontam que houve uma perda de mais de $878 \mathrm{~km}^{2}$ de cobertura vegetal correspondendo cerca de $12 \%$ de perda que veio em decorrência das atividades antrópicas que ocorreram em Moju, principalmente, em relação à agricultura com os cultivos de dendê, mandioca, coco e à pecuária com as áreas de pastagens, pois, juntos apresentam um aumento de mais de $70 \%$ que equivalem a $1.037,0938 \mathrm{~km}^{2}$. Ressalta-se que também na região, são comuns as práticas ilegais de retirada da madeira para comercialização e atividades de carvoarias que abastecem as indústrias da região. Associado a essas transformações, está o fato do município, em 2010, ter entrado para a lista dos municípios que mais desmatam o bioma amazônico (Cardoso et al., 2012).

Silva (2016) ressalta que a crescente dinâmica do desmatamento na região é condicionada às diversas atividades econômicas presentes no município. A expansão dos monocultivos associados à criação de gado e ao cultivo de dendê, por meio de empresas financiadas pelo capital nacional e internacional, tem condicionado ao município, um ciclo econômico e de migração inter e intra regional. Se por um lado o desmatamento promove consequências negativas ao meio natural e social, por outro lado ele implica positivamente ao surgimento e à condução de novas atividades econômicas.

A partir de análises realizadas nessa pesquisa e do suporte de outros estudos relacionados ao uso e cobertura da terra no município, pode-se inferir que apesar de inicialmente a expansão dos monocultivos em Moju ter se estabilizado em áreas antigamente ocupadas pela pecuária e por pequenos cultivos, nota-se uma relevante alteração na cobertura vegetal da região, principalmente em área de florestas secundárias em fase de regeneração, evidenciando uma nova tendência produtiva relacionada ao monocultivo de dendezeiro no município.

\section{Conclusão}

Constatou-se que o desenvolvimento econômico do município de Moju segue o padrão de desenvolvimento dos municípios amazônicos, onde ocorre a diminuição das áreas florestais para a expansão de suas atividades produtivas, como os cultivos de dendê, sendo este um dos principais indutores do desflorestamento da área do município. Sendo assim, pode-se destacar que a dinâmica do uso e cobertura de Moju está baseada na consolidação dos produtos agropecuários como uma das variáveis econômicas da localidade.

Nessa perspectiva, a compreensão sobre as tendências da dinâmica do uso e cobertura do solo, é de fundamental importância para promover subsídios capazes de exterminar ou minimizar impactos socioeconômicos e ambientais em municípios amazônicos, uma vez que os mesmos têm sofrido elevadas pressões quanto ás atividades econômicas consolidadas ao longo dos anos. Logo, o monitoramento ambiental com o auxílio de ferramentas do geoprocessamento, é essencial para combater o avanço e as consequências dos principais problemas relacionados às modificações do uso e cobertura da terra.

O uso do Google Earth Engine (GEE) apresenta-se como uma nova e potente ferramenta para análises de uso e cobertura da terra, uma vez que permite adquirir dados gratuitos e de fácil acesso, sendo primordial para o fornecimento de dados ambientais.

Portanto, por meio desse estudo, podem-se realizar outras pesquisas relacionadas às mudanças de uso e cobertura da terra, como a avaliação dessas modificações provenientes de atividades econômicas, como a mineração e o extrativismo vegetal, e a análise da dinâmica da Integração Lavoura-Pecuária-Floresta (ILPF) em áreas de restauração em municípios amazônicos.

\section{Agradecimentos}

Os autores agradecem à Universidade Federal Rural da Amazônia (UFRA) e ao Programa de Pós Graduação em Ciências Ambientais 
(PPGCA) da Universidade do Estado do Pará (UEPA).

\section{Referências}

Almeida, A.S., Vieira, I.C.G., 2014. Conflitos no uso da terra em áreas de preservação permanente em um polo de produção de biodiesel no estado do Pará. Revista Ambiente e Água 9(3).

Cardoso, A.S., Vieira, I.C.G., Almeida, A.S., Ferreira, A.E.D.M., Lameira, W.J.D.M., 2012. Dinâmica socioambiental no município do Moju em tempos de biodiesel. 2012. VI Encontro Nacional da Anppas, Belém-PA.

Cardoso, A.S., Toledo, P.M., Vieira, I.C.G., 2016. Barômetro Da Sustentabilidade Aplicado ao Município de Moju, Estado do Pará. Revista Brasileira de Gestão e Desenvolvimento Regional 12(1), 234-263.

Bastos, A.P., Almeida, O., Castro, E.D., Marin, R., Pimentel, M., Rivero, S., Bruzeke, F., 2010. Economia e sociedade na região do Tocantins, Pará. Papers, Belém: NAEA, 1-28.

Brasil., 2011. Ministério do Meio Ambiente. Dispõe sobre a lista de municípios situados no Bioma Amazônia onde incidem ações prioritárias de prevenção, monitoramento e controle do desmatamento ilegal.

Silva, F.L.D., 2016. Dimensões de uso e cobertura da terra: uma análise voltada a classificar municípios do estado do Pará e promover serviços ecossistêmicos no município de MojuPA.

Gollnow F., Lakes, T., 2014. Policy change, land use, and agriculture: The case of soy production and cattle ranching in Brazil, 2001 e 2012. Applied Geography, 55, 203-211.

Gorelick, N.,Hancher, M., Dixon, M., Ilyushchenko, S., Thau, D., Moore, R., 2017. Google Earth Engine: Planetary-scale geospatial analysis for everyone. Remote Sensing of Environment, 202, 18-27.

IBGE. Instituto Brasileiro de Geografia e Estatística. Censo Demográfico. 2015. Acesso em 25 de maio de 2020. Disponível em: https://www.ibge.gov.br/estatisticas/multidomi nio/cultura-recreacao-e-esporte/9662-censodemografico-2010.html?=\&t=o-que-
IBGE. Instituto Brasileiro de Geografia e Estatística. Produção Agrícola Municipal. 2018. Acesso em 30 de maio de 2020. Disponível em: https://sidra.ibge.gov.br/tabela/5457.

Mertens, B., Poccard-Chapuis, R., Piketty, MG, Lacques, AE e Venturieri, A., 2002. Crossing spatial analyses and livestock economics to understand deforestation processes in the Brazilian Amazon: the case of São Félix do Xingu in South Pará. Agricultural Economics, 27(3), 269-294, 2002.

Modesto Junior, M.S., Andrade, A.C.S., Alves, R.N.B., 2008. Transferência de tecnologia pelo método treino e visita para agricultura familiar no Município de Moju, Estado do Pará. In: Congresso da Associação Brasileira das Instituições de Pesquisa Tecnológica. Campina Grande. Os desníveis regionais e a inovação no Brasil: os desafios para as instituições de pesquisa tecnológica: resumos. Brasília, DF.

Nepstad, D., 2000. Avança Brasil: Os custos ambientais para a Amazônia. Belém: Gráfica e Editora Alves.

Pimenta, L., Beltrão, N.,Gemaque, A., Pontes, A., 2018. Dinâmica do uso e cobertura da terra em municípios prioritários: uma análise no município de Moju, Pará no período de 2008 a 2014. GOT, Revista de Geografia e Ordenamento do Território.

Steffen, W., Sanderson, R.A., Tyson, P.D., Jäger, J., Matson, P.A., Moore III, B., Oldfield, F., Richardson, K., Schellnhuber, H.-J., Turner, B.L., Wasson, R.J., 2004. Global change and the Earth system: a planet under pressure. Berlin, Germany: Springer.

Santos, E.D.V.D., Leite, G.C.D.S., Vieira, D.C.D.M., Cruz Filho, J., 2017. A dendeicultura no município de Moju: Transformações socioespaciais e dinâmica migratório. Revista Eletrônica Geoaraguaia, Barra do Garças-MT 7(2), 48-67.

Vale, J.R.B., 2019. Análise da dinâmica do uso e cobertura da terra nas áreas desflorestadas do Estado do Pará por meio da Plataforma Google Earth Engine, Belém-PA. 